\title{
Phantom hand and wrist movements in upper limb amputees are slow but naturally controlled movements
}

Jozina B. De Graaf ${ }^{{ }^{*}}$, Nathanaël Jarrassé ${ }^{2}$, Caroline Nicol ${ }^{1}$, Amélie Touillet ${ }^{3}$, Thelma Coyle ${ }^{1}$, Luc Maynard $^{4}$, Noël Martinet ${ }^{3}$, Jean Paysant ${ }^{3}$

${ }^{1}$ Institute of Movement Sciences, UMR 7287 - CNRS \& Aix-Marseille University, Marseille, France.

${ }^{2}$ CNRS, UMR 7222, ISIR / INSERM, U1150 Agathe-ISIR, Sorbonne University, UPMC Univ Paris 06, Paris, France.

${ }^{3}$ Louis Pierquin Centre of the Regional Institute of Rehabilitation, Nancy, France.

${ }^{4}$ Centre for Functional Rehabilitation of Valmante, Marseille, France.

\section{Correspondence:}

Dr. Jozina De Graaf

Institute of Movement Sciences

UMR 7287 -Aix-Marseille University - CNRS

Faculty of Sport Sciences

163, Avenue de Luminy, CP 910

13288 Marseille cedex 9

France

jozina.de-graaf@univ-amu.fr

Running title: Quantification of phantom hand mobility 


\begin{abstract}
After limb amputation, patients often wake up with a vivid perception of the presence of the missing limb, called "phantom limb". Phantom limbs have mostly been studied with respect to pain sensation. But patients can experience many other phantom sensations, including voluntary movements. The goal of the present study was to quantify phantom movement kinematics and relate these to intact limb kinematics and to the time elapsed since amputation. Six upper arm and two forearm amputees with various delays since amputation (6 months to 32 years) performed phantom finger, hand and wrist movements at self-chosen comfortable velocities. The kinematics of the phantom movements was indirectly obtained via the intact limb that synchronously mimicked the phantom limb movements, using a Cyberglove $^{\circledR}$ for measuring finger movements and an inertial measurement unit for wrist movements. The results show that the execution of phantom movements is perceived as "natural" but effortful. The types of phantom movements that can be performed are variable between the patients but they could all perform thumb flexion/extension and global hand opening/closure. Finger extension movements appeared to be $24 \%$ faster than finger extension movements. Neither the number of types of phantom movements that can be executed nor the kinematic characteristics were related to the elapsed time since amputation, highlighting the persistence of post-amputation neural adaptation. We hypothesize that the perceived slowness of phantom movements is related to altered proprioceptive feedback that cannot be recalibrated by the lack of visual feedback during phantom movement execution.
\end{abstract}

Keywords: Voluntary phantom movements; amplitude; velocity; neuroplasticity; evolution over time; movement feedback.

\title{
1. Introduction
}

Phantom limbs, reported by $90-98 \%$ of amputees (Ramachandran and Hirstein, 1998), have been recognized for a long time, first from religious images dating at least 600 years (Schott, 2014), and later from medical descriptions in the nineteenth and twentieth centuries (e.g., Mitchell, 1891; Henderson and Smyth, 1948). These early reports perfectly describe, albeit in a qualitative way, most of the currently known phantom limb phenomena. Yet, despite the scientific and medical acceptance of phantom phenomena, phantom limbs are a social problem. Indeed, many patients and their family still consider phantoms as "imaginary" and a "sign of non-acceptance of the limb loss", and their existence is often ignored by them. Even the medical community usually only considers phantom sensations with respect to pain. Phantom limbs have therefore mostly been studied with respect to associated pain sensations (e.g., Kooijman et al., 2000) whereas non-painful phantom limbs have attracted much less attention. Still, even when ignoring their phantom limb, many patients can perceive it for decades after the amputation (Ramachandran and Hirstein, 1998); it seems that phantom limbs reveal mechanisms of long-lasting neural plasticity in the adult brain. 
Following the surgery, in $75 \%$ of the cases, phantom sensations can be felt immediately after waking up (Xerri, 2003) and remain pronounced for the first 6 months (Carlen et al, 1978), but they can also appear several years later (Henderson and Smyth, 1948) and stay for a lifetime. Although the phantom limbs can be perceived as normal in terms of morphology and position, many patients report abnormal or deformed phantom limbs in terms of length, proportion, posture... which may change in time (André et al., 2001). Our own observations showed phenomena such as perception of the phantom hand at its "right place" (i.e., at the same distance from the most distal remaining articulation as for the intact arm) but with a complete loss of perception of the forearm and elbow, which implies that the phantom hand is not felt as attached to the residual limb but as "floating in the air".

Much less known is the fact that many patients describe the capacity of voluntary mobilization of their phantom limb. In our recent epidemiological study, at the time of the interview of almost fifty upper-limb amputees, $85 \%$ reported having a mobile phantom limb and only three patients declared never having been able to produce any voluntary limb movement (Touillet et al., submitted). Since phantom movements cannot be observed, they are often confounded with motor imagery of the lost limb. Yet, recent studies show that movement control of the phantom limb is different from motor imagery. Indeed, it has been shown that phantom movement execution is generally slower than intact limb execution whereas the time to imagine a movement is similar for both the phantom and the intact limb (Raffin et al., 2012a). Also, cortical activation involved in phantom movement execution differs from that involved in phantom movement imagery but is similar to that of intact limb movement execution (Raffin et al., 2012b). In addition, muscle activity is always present in the residual limb during phantom movement execution, but imagery may be performed without (Raffin et al., 2012a). Moreover, these muscle activation patterns are not random but systematically vary with the type of phantom movement (Gagné et al., 2009; Reilly et al., 2006). Finally, phantom hand movement sensations can be evoked by transcranial magnetic stimulation (TMS) of the hand area of the primary motor cortex (Gagné et al., 2011; Mercier et al., 2006). Altogether, these results strongly suggest that phantom limb mobility results from specific motor commands sent from the cortex to the missing limb, which, since the target of the motor commands is lost, project on the remaining residual limb muscles.

The few studies having explored the kinematics of phantom movements generally agree that phantom movements are slower and/or smaller in amplitude (Gagné et al., 2009; Henderson and Smyth, 1948; Reilly et al., 2006). Henderson and Smith (1948) observed that, after amputation, finger flexion movements persist longer than finger extension movements and that the movement amplitude depends on the level of amputation (forearm versus upper arm). Yet, to our knowledge, these observations were neither further quantified nor mentioned by other studies; the few studies on the kinematics of phantom movements were based on the whole cycle of a given movement in above-elbow amputees (Gagné et al., 2009; Reilly et al., 2006). Moreover, previous studies investigated some pre-selected phantom movements rather than the whole panel for a given patient. A deeper insight in these phenomena will shed more light on the neurophysiological mechanisms underlying phantom movement control. Therefore, the goal of the present study was to explore the kinematic characteristics of a large 
panel of voluntary phantom movements in upper arm and forearm amputees. Firstly, we investigated whether the panel of phantom movements reveals the possibility for combined finger movements, such as the (for humans important and well developed) precision grip. Second, as phantom movements differ from motor imagery (Raffin et al., 2012b), we analyzed whether they are influenced by fatigue as do intact limb movements. Third, given that flexion movements were found in the literature to disappear earlier than extension movements (e.g., Henderson and Smith, 1948), we investigated whether these latter are easier and faster. Finally, we explored whether the number of different types of phantom movements, as well as their amplitude and velocity, diminish in time.

\section{Experimental Procedures}

\subsection{Participants}

Eight patients (aged 27 to 76 years, one woman) were selected to participate in the study. The selection was based on the reported presence of a mobile phantom limb, the ability to imitate the phantom movements in real time with the intact limb, and on the availability of the patient during the period of recording. We excluded patients that experienced phantom limb pain during movement execution as it has been shown that this modifies phantom movement kinematics and limits their movement capacity (e.g., Gagné et al., 2009). Table I resumes demographic data. Six of the patients were upper arm amputees; two patients had a forearm amputation. For all eight patients, the amputation was of traumatic origin. The elapsed time since amputation varied between 6 and 392 months.

According to the Declaration of Helsinki, all procedures were carried out with the adequate understanding and written consent of the patients. A qualitative phantom movement protocol was already included in their medical follow-up; the addition of quantification by recording with a Cyberglove ${ }^{\circledR}$ was approved by the Local Ethical Committees of the involved rehabilitation centers.

Table 1. Demographic data concerning the 8 recorded patients. $M=$ male; $F=$ female; Elapsed time $=$ time since amputation. "Prosthesis" indicates the type of prosthesis the patient usually wears.

\begin{tabular}{|c|c|c|c|c|c|c|}
\hline Patient & $\begin{array}{c}\text { Age } \\
\text { (years) }\end{array}$ & Gender & Amputation & $\begin{array}{c}\text { Elapsed } \\
\text { time } \\
\text { (months) }\end{array}$ & $\begin{array}{c}\text { Amputated } \\
\text { side/dominant } \\
\text { side }\end{array}$ & Prosthesis \\
\hline P1 & 31 & M & Upper arm & 6 & Right/Left & Myoelectric \\
P2 & 52 & M & Upper arm & 392 & Left/Right & Esthetical \\
P3 & 76 & F & Upper arm & 165 & Right/Right & Myoelectric \\
P4 & 54 & M & Upper arm & 34 & Left/Left & Esthetical \\
P5 & 42 & M & Upper arm & 58 & Right/Right & Myoelectric \\
P6 & 33 & M & Upper arm & 43 & Right/Right & Myoelectric \\
P7 & 27 & M & Forearm & 82 & Left/Right & Myoelectric \\
P8 & 33 & M & Forearm & 78 & $\begin{array}{c}\text { Right/ } \\
\text { Ambidextrous }\end{array}$ & Not anymore \\
\hline
\end{tabular}




\subsection{Protocol}

The recordings were performed in the rehabilitation centers in the presence of a medical doctor. For each patient, the recording session started by identifying the different types of phantom movements the patient was able to execute. Over the whole group of patients, different types of phantom movements appeared to be possible, ranging from individual finger movements to multi-segment movements such as precision grip and global hand opening and closure. The self-reported effort required for the execution of each of them determined the type and order of the tested movements, from the easiest (less effortful) to the hardest. Each possible type of phantom movement was tested in a sequence of ten to twelve cyclic repetitions at a self-chosen comfortable movement velocity. The patient was instructed to synchronously mimic the phantom movements with the intact limb. Each sequence was followed by a resting period of several minutes. The experimenter counted the cycles and verbally indicated the beginning and end of each sequence. This could occur earlier than after ten cycles when the patient could not move the phantom limb anymore (generally due to fatigue). At the end of each sequence, the patient reported the experienced difficulty associated with the phantom movement execution (fatigue or residual limb pain) and the quality of the mimicking with the intact limb. If the patient estimated that the mimicking was not correctly done, the sequence was repeated. This, however, occurred very rarely.

\subsection{Recording}

A Cyberglove ${ }^{\circledR}$ II was used to record the angular postures of the five fingers from the intact (left or right) hand while mimicking the phantom hand movements. The Cyberglove ${ }^{\circledR}$ allows an accurate and complete capture of finger movements with a precision below $1^{\circ}$. This device relies on the use of a large number of piezoelectric sensors which are sewn inside the elastic glove at each joint (Fig. 1A), allowing the capture of complex hand movements. Although the flexible glove is adaptable to many hand sizes, it has to be calibrated for each subject in order to get representative measurements independent of the subject's hand morphology. We therefore developed a simple and fast calibration method derived from the procedure introduced by Huenerfauth and Lu (2010) which relies on the classical use of keypostures. For each patient, calibration was performed once before the phantom movement execution task and the calibration data were kept and used during the entire session since the subjects did not remove the glove until the end of the session.

In order to track wrist movements (none of the six upper arm amputees appeared to be able to perform phantom elbow movements), an additional Inertial Measurement Unit (IMU, nine degree-of-freedom Sensor Stick from Sparkfun ${ }^{\circledR}$ ) was attached to the top of the glove, allowing a precise reconstruction of the hand orientations in space (thanks to a dedicated sensor fusion algorithm calculating IMU orientations based on the accelerometer, gyroscope and magnetometer information) and thus of the range of the patients' wrist movements (flexion/extension, pronation/supination, ulnar/radial inclination). A dedicated interface was developed in $\mathrm{C}++$ with OpenGl and the use of multiple threads in order to ease the calibration phase for the experimenter and the patient (thanks to a real-time visualization function) and to 
perform precise synchronized $100 \mathrm{~Hz}$ acquisition of both the IMU and Cyberglove ${ }^{\circledR}$ data. The whole recording session, lasting for about 45 minutes, was also videotaped.
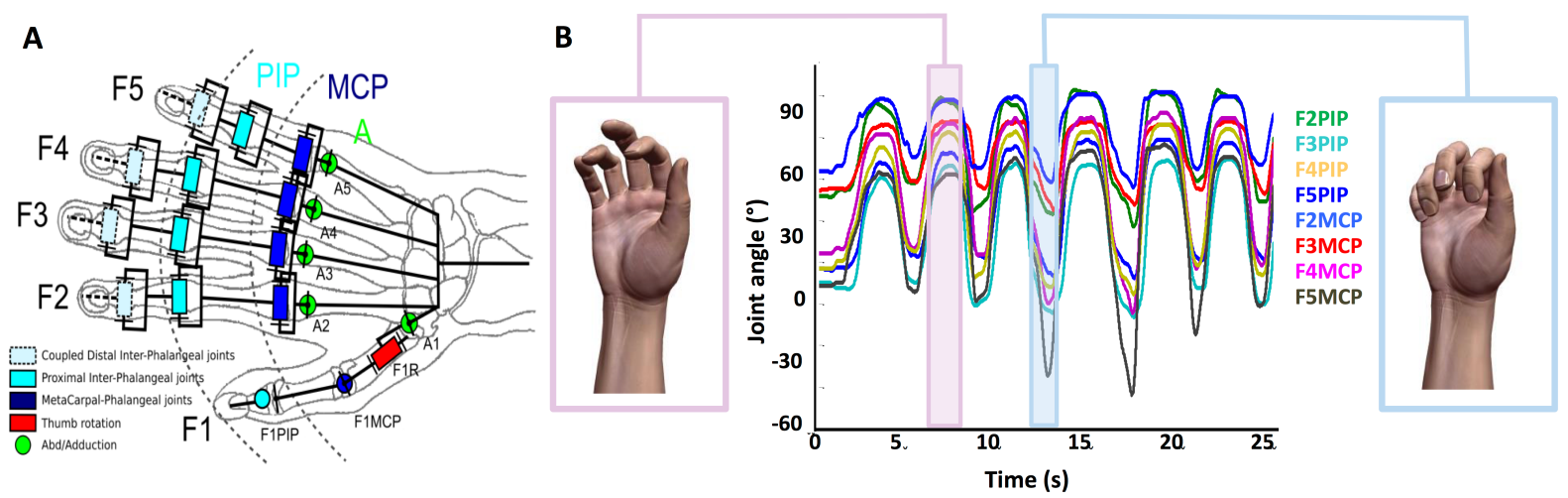

Fig. 1. (A) Schematic drawing of the fifteen degree-of-freedom kinematic model of the right hand Cyberglove ${ }^{\circledR}$ II. Of the eighteen sensors on the glove, only fifteen were used. F1 to F5 identify respectively the thumb, the index, the major, the ring and the pink. $\mathrm{A}(\mathrm{i}, \mathrm{i}+1)$ (with $\mathrm{i} \in\{1, . ., 4\}$ ) represents the abduction and adduction joint between a finger $i$ and the following one $(i+1)$. $\mathrm{PIP}=$ proximal inter-phalangeal joints; $\mathrm{MCP}=$ metacarpal-phalangeal joints. The piezoelectric sensors are sewn inside the elastic glove at each joint level. All these joints were measured during the experiments. Nevertheless, because of the glove technology, "coupled abductions" of the fingers were recorded (A12, A23, A34, A45) instead of abduction (A1, A2, A4, A4, A5) of each finger. The distal phalangeal joints are not considered as they are coupled with and proportional to the proximal phalangeal joints. (B) Reconstructed 3D capture of the left imitating (intact) hand, as well as the angles of the joints of fingers 2 to 5 (in different colors) of patient P6 during the first 25 $\mathrm{s}$ of hand opening and closing, mimicking the opening and closing of the phantom hand.

\subsection{Analysis}

The Cyberglove ${ }^{\circledR}$ data were filtered with a fourth-order low-pass Butterworth filter (cutoff frequency $2.5 \mathrm{~Hz}$ ). For the cycles in which the amplitude and frequency became reduced due to fatigue, we excluded the last cycles based on the patient's report and visual inspection of the video. Then, to quantify the phantom movements, kinematic data was obtained by analyzing the channels involved in the executed phantom movement from either the Cyberglove ${ }^{\circledR}$ or the IMU. For the individual finger movements, we averaged the values obtained for the two (for fingers 2 to 5) or three (for finger 1) recorded joints. For the precision grip we averaged the values obtained for the three joints of finger 1 and the two joints of finger 2 (or fingers 1 and 5 for P5 who performed an opposition of thumb and pinkie). For the hand opening and closing, we averaged over all eleven recorded finger joints to have an estimation of global hand movement. For the wrist movements, we analyzed the IMU channel for which the angular variation was highest.

For each patient and each type of phantom movement, we determined the number of executed cycles. Then, for each cycle, we determined duration, total amplitude of the movement range as well as angular peak velocity (Vpeak) of both phases of the cycle (i.e., separating flexion from extension, pronation from supination, etc). In order to test whether the mean cycle duration and the number of times that a patient could repeat a given type of 
phantom movement were related to the amplitude of the movement, Pearson tests for linear correlation were applied to the data.

We compared peak velocities of finger, precision grip and hand movements between the flexion and extension phases. Note that for wrist movements it is difficult to analyze the results in such a way since different wrist movements are around different axes and therefore cannot be classified in two classes as can be done for finger/hand movements. The peak velocities being highly variable between participants and between the different types of phantom movements, a statistical difference in Vpeak between extension and flexion phases could not be obtained by directly comparing the obtained values. Therefore, for each type of phantom movement and each participant, we plotted the Vpeak obtained during extension as a function of that obtained during flexion movements. A Pearson test for linear correlation was applied to the data, and as the correlation was significant, the linear equation coefficient was determined. If both phases are executed at similar velocities, the slope of their linear relation will be 1 . If extension is executed at higher velocities, the slope will be above 1; if flexion is executed at higher velocities, the slope will be below 1 . So, the linear equation coefficient gives information about the relative velocity of extension with respect to that of flexion movements.

In order to explore how phantom movements evolve in time, we determined whether (1) the number of different types of phantom movements the patients could perform, and (2) the amplitude and angular peak velocity of phantom movements, were related to the time elapsed since amputation. For this latter point, we determined for each patient the amplitude as well as the peak velocity averaged over the two types of phantom movements that all patients had in common: flexion/extension of the thumb and opening/closure of the hand. Then, we studied the relation between the three variables and the elapsed time with Pearson tests for linear correlation.

The whole kinematic analysis was done with help of a custom made Matlab (version 8.0.0.783) function. All statistical testing was performed in Statistica ${ }^{\circledR} 7.1$ with the significance threshold fixed at 0.05 .

\section{Results}

Due to a technical problem during recording, the Cyberglove data of P4 could not be analyzed. We used the video recording of this patient to identify the types of phantom movements executed and to count the number of cycles; however, the quantification of the phantom movement kinematics was not possible. Therefore, the kinematic results are based on the data of seven patients.

\subsection{Global description of phantom movements}

It appeared that, for each patient and each phantom movement, the amplitudes were quite stable over a sequence of cycles. Fig. 1B shows an extract of the finger joint angles of the mimicking hand as a function of time during cyclic global opening and closing of the phantom hand for patient P6 as well as the corresponding reconstructed 3D capture at maximal opening and closure. It can be seen that the movement amplitude is stable throughout the $25 \mathrm{~s}$ shown here. Similar stability was seen for all patients and for all types of 
phantom movements. This was confirmed by the grand average standard error (SE) of the amplitude (i.e., first calculated for each type of phantom movement and averaged over all types of phantom movements per patient, and then averaged over all patients) which was only $1.4 \pm 0.3^{\circ}$ (mean SE $\pm \mathrm{SE}$ ).

Table 2 shows the number of cycles the patients were able to perform, the mean cycle duration and the total amplitude for each type of phantom movement performed by a least one of the eight patients. All patients were able to perform several types of phantom finger and hand movements. All eight patients were able to flex and extend their thumb (finger 1), and to open and close their whole phantom hand. All upper-arm and one of the forearm amputees were able to open and close the precision grip (i.e., thumb/index opposition, except for P5 who performed a thumb/pinkie opposition). Six patients were able to individually mobilize the index (finger 2) and the little finger (finger 5). Since phantom fingers 3 and 4 often moved together, the results for these fingers were merged (from here-on called "fingers 3-4"). Only one patient (P2) was able to individually mobilize fingers 3 and 4; for this patient we have arbitrarily chosen the results of finger 3 to be shown in Table II. Concerning wrist movements, five of the patients were able to perform flexion and extension movements and four patients could execute rotations and/or inclinations. It should be noted that none of the six upper arm amputees in our study was able to make phantom elbow movements as they either perceived their hand directly attached to the residual limb (P2, P4) or the phantom elbow joint was not perceived even if their hand was not felt directly attached to the residual limb (P5, P6). The two participants perceiving their elbow (P1 and P3) reported that the attempts to make it move did not result in any for-them-detectible movement and rapidly gave rise to an important fatigue.

Table 2. Number of cycles performed, mean cycle duration and mean cycle amplitude obtained for each type of phantom movement and for each patient. Fingers 3 and 4 often moved together and were therefore merged ("finger 3-4"). Only one patient (P2) was able to mobilize each of fingers 3 and 4 individually; for this patient the results of finger 3 were arbitrarily chosen to be shown. Empty cases indicate that the patient was not able to make the given phantom movement. For P4 no kinematic data was available. $\mathrm{N}=$ Number of performed cycles; $\mathrm{D}=$ Cycle duration averaged over all cycles in the sequence (in s); $\mathrm{A}=$ Cycle amplitude averaged over all cycles in the sequence $\left(\right.$ in $\left.^{\circ}\right)$.

\begin{tabular}{|c|c|c|c|c|c|c|c|c|c|c|c|c|c|c|c|c|c|c|c|c|c|c|c|c|c|c|c|}
\hline & \multicolumn{3}{|c|}{$\begin{array}{l}\text { Fles/Est } \\
\text { finger I }\end{array}$} & \multicolumn{3}{|c|}{$\begin{array}{l}\text { Fles/Est } \\
\text { finger } 2\end{array}$} & \multicolumn{3}{|c|}{$\begin{array}{c}\text { Flex/Ext } \\
\text { fingers 3-4 }\end{array}$} & \multicolumn{3}{|c|}{$\begin{array}{l}\text { Flesest } \\
\text { finger } 5\end{array}$} & \multicolumn{3}{|c|}{$\begin{array}{l}\text { Open/Close } \\
\text { precision grip }\end{array}$} & \multicolumn{3}{|c|}{$\begin{array}{c}\text { OpenClose } \\
\text { land }\end{array}$} & \multicolumn{3}{|c|}{$\begin{array}{c}\text { Fles/Est } \\
\text { wrist }\end{array}$} & \multicolumn{3}{|c|}{$\begin{array}{c}\text { Retation } \\
\text { wrist }\end{array}$} & \multicolumn{3}{|c|}{$\begin{array}{c}\text { Inclination } \\
\text { wrist }\end{array}$} \\
\hline & $N$ & $D(s)$ & & $\mathrm{N}$ & D (s) & A(") & $\mathrm{N}$ & $D$ (s) & $M C I$ & $N$ & $\begin{array}{l}\mathrm{D} \\
\text { (0) }\end{array}$ & $A C I$ & N & $\begin{array}{c}D \\
|x|\end{array}$ & $M(I)$ & $\mathrm{N}$ & $\mathbf{D}$ (s) & $M$ & $N$ & $\begin{array}{l}\text { D } \\
\text { (is) }\end{array}$ & $\begin{array}{c}M P \\
1\end{array}$ & N & $\begin{array}{l}\text { D } \\
\text { (0) }\end{array}$ & $M^{\prime \prime}$ & $N$ & $\begin{array}{l}\mathrm{D} \\
\langle\mathrm{x}\rangle \\
\end{array}$ & S \\
\hline PI & 6 & 19 & 208 & 9 & 3.6 & 56.6 & 4 & 5.6 & 48.7 & 10 & 1.8 & $\begin{array}{c}46 . \\
6\end{array}$ & 9 & 2.8 & $\begin{array}{c}18 . \\
18\end{array}$ & 7 & 46 & 43.4 & 11 & 3.5 & 34 & & & & 4 & 3.7 & 48 \\
\hline$P 2$ & 10 & 20 & 49 & 12 & 1.3 & 2.4 & 11 & 20 & 2.7 & 10 & 1.8 & 4.0 & 10 & 1.3 & 1.0 & 5 & 4.3 & 4.9 & 10 & 2.3 & 3 & 12 & 2.4 & 6 & 8 & 6.5 & 7 \\
\hline P3 & 12 & 19 & 16.1 & 9 & 1.7 & 24.2 & 8 & 21 & 42.1 & 10 & 2.1 & $\begin{array}{c}20 . \\
5\end{array}$ & 7 & 2.7 & 9.3 & 10 & 23 & 27.5 & & & & 11 & 2.8 & 128 & 11 & 1.9 & 28 \\
\hline$P_{4}$ & 5 & & & & & & & & & & & & 2 & & & 3 & & & 3 & & & & & & & & \\
\hline P5 & 6 & 15 & 50 & & & & & & & & & & 5 & 2.5 & 7.8 & 5 & 3.4 & 8.5 & 5 & 1.7 & 3 & & & & & & \\
\hline Po & 9 & 28 & 17.4 & 11 & 2.9 & 25.1 & 11 & 22 & 26.7 & 12 & 3.2 & 34. & 7 & 2.6 & 8.5 & 13 & 32 & 33.5 & & & & & & & & & \\
\hline $\mathbf{P 7}$ & 11 & 09 & 5.7 & 10 & 1.1 & 21.0 & 17 & 10 & 19.4 & 12 & 0.9 & $\begin{array}{c}20 . \\
4\end{array}$ & 9 & 1.3 & 9.5 & 11 & 18 & 49.8 & 9 & 1.2 & 51 & 14 & 1.3 & 99 & & & \\
\hline P8 & 9 & 78 & 13.7 & 9 & 6.4 & 51.0 & 6 & 60 & 4.0 & 11 & 6.2 & $\begin{array}{c}74 . \\
6 .\end{array}$ & & & & 10 & 10.1 & 21.7 & & & & & & & & & \\
\hline
\end{tabular}

\subsection{Influence of fatigue of phantom movement execution}

The patients reported that some movements were more difficult to execute than others. In this case, the cycle duration became longer and/or the patient was not able to continue for 
ten cycles. However, the type of phantom movements that was considered as difficult varied between the patients. For instance, global hand opening/closing was difficult for patient P2 who performed only 5 cycles of rather long durations ( $4.3 \mathrm{~s}$ on the average) and then stopped because of fatigue. The same patient performed 10 cycles at $1.3 \mathrm{~s}$ per cycle of precision grip opening/closing whereas another patient (P6) felt no difficulty in opening/closing the global hand for thirteen cycles, but stopped after seven precision grip opening/closing movements because of fatigue. Patient P8 (a forearm amputee) appeared to be rather a-typical with respect to this general finding. This patient performed about ten cycles with long cycle durations for each type of phantom movement without experiencing fatigue. So, cycle duration does not always reflect movement execution effort. Despite the inter-patient variability, we found a small but significant negative correlation between the number of executed movement cycles and mean cycle duration $\left(\mathrm{r}^{2}=0.43, \mathrm{p}<0.001\right)$. We found no correlation between movement amplitude and either cycle duration $\left(r^{2}=0.08, p>0.05\right)$ or number of cycles $\left(r^{2}=0.02, p>0.4\right)$, which is not surprising given the results shown in Table II. For instance, patient P2 easily executed ten short cycles $(2.3 \mathrm{~s})$ of flexion/extension of the wrist with an amplitude of only $3^{\circ}$ whereas patient P8 performed eleven long cycles $(6.2 \mathrm{~s})$ of flexion/extension of finger 5 with an amplitude of $149^{\circ}$ and patient P7 performed ten very short cycles $(1.8 \mathrm{~s})$ of hand opening/closure with an amplitude of $110^{\circ}$. Even for patient S8 (very slow movements over long durations), taking into account each phase of each cycle, a Pearson test for linear regression did not show a linear relation between duration and amplitude $\left(r^{2}=0.001, p>0.7\right)$.

Regarding the reported phantom sensations related to fatigue, all patients mentioned that fatigue induced a feeling of having their phantom hand "bound" or "restrained in a tube", with an "impossibility to move their fingers (hand, wrist), as if they are blocked". Some patients also mentioned that their phantom sensations (like pricking or tinkling) in the moving phantom limb increased with increasing effort; others gradually lost their phantom sensations (which came back as soon as the patient stopped trying to move). In all cases, fatigue induced hyperhidrosis. Interestingly, several patients spontaneously mentioned that the effort they experienced during the execution of some phantom movements was due to a "lack of exercise" and were convinced that practicing could reduce the level of effort. A typical example of this is the execution of phantom pinkie movements of P6, who easily performed twelve cycles within $40 \mathrm{~s}$. This patient mentioned that, regularly during the day, he performed these movements to relieve himself from pain in the residual limb. Another example is patient P4 (for whom we do not have any exploitable kinematic data) who did not practice phantom movements for years ("because phantom movements are not useful"): although the movements were still effortful (as suggested in the video recordings by the observed small number of cycles he performed very slowly), he reported having increased the number of cycles he could perform by practicing only some days before the recording session.

\subsection{Kinematics of phantom movements}

Fig. 2 shows the amplitude (Fig. 2A) and peak velocity (Fig. 2B) of the different types of phantom movements, averaged over all patients. The whisker diagrams are based on the mean values obtained for each participant. The SE and the total interval of the values show the high inter-participant variability that could already be observed in Table 2. For the results 
concerning the peak velocities of the phantom movements, we separated the two phases of each cycle, i.e., flexion and extension for the fingers, opening and closing for the precision grip and global hand, and flexion and extension, pronation and supination and also radial and ulnar inclination for the wrist. For the finger, precision grip and hand movements, the mean peak velocity for extension/opening movements (in dark grey in the figure) tends to be systematically higher than that for flexion/closing movements. This observation is confirmed in Fig. 2C showing, for each participant and each type of phantom finger and hand movement, the peak velocity during the extension phase as a function of that of the flexion phase. The correlation between the two variables being significant $(\mathrm{p}<0.001)$, the linear regression equation shows that extension movements are $24 \%$ faster than the flexion movements.

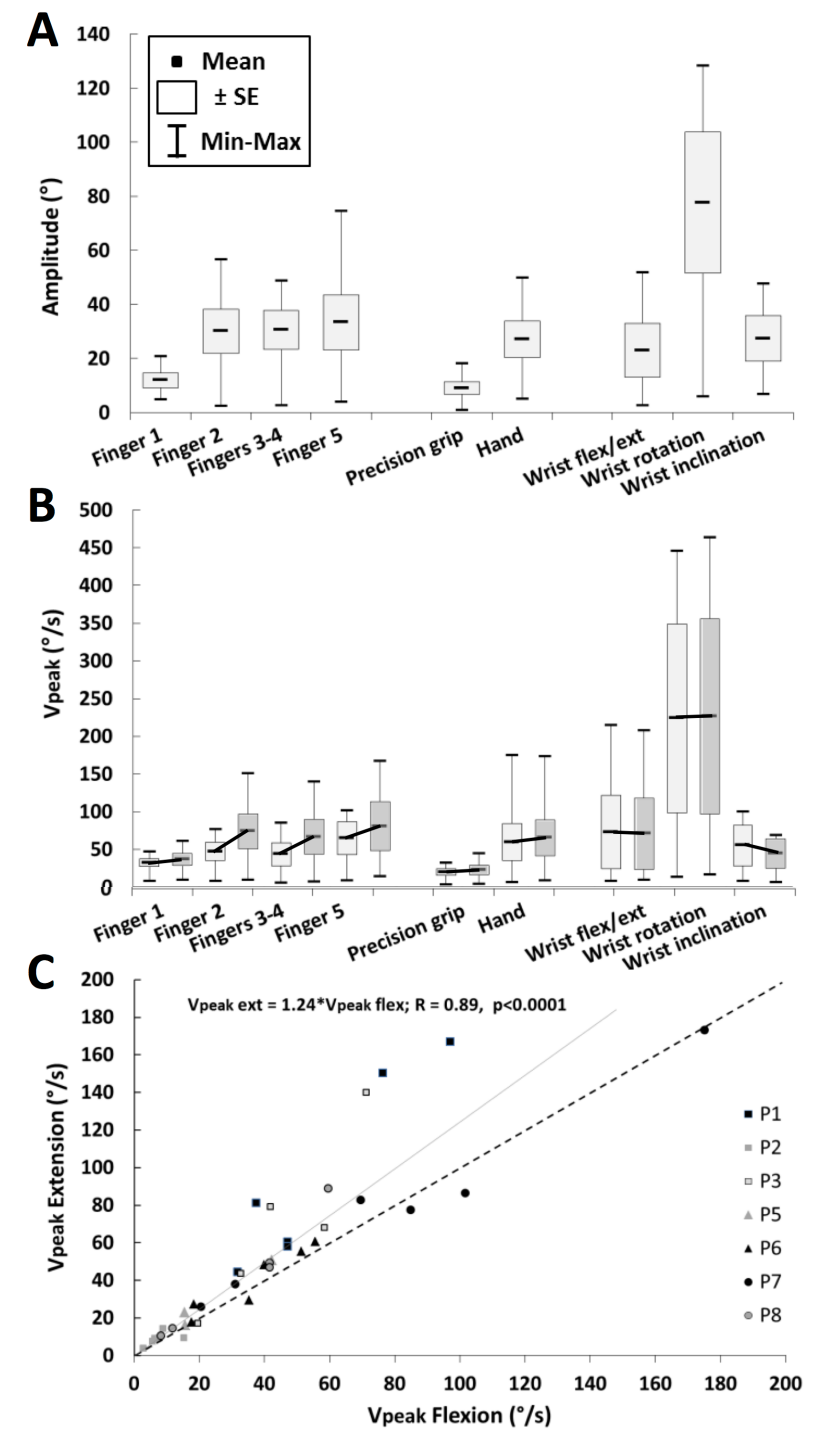

Fig. 2. Results based on the values obtained for each patient averaged over a whole sequence of the concerned types of cyclic phantom movements. A. Amplitude (in degrees) (values of Table II averaged over all patients). B. Corresponding mean peak velocities (Vpeak, \%s), separated for the two phases of each type of phantom movement, i.e., for flexion (light grey) respectively extension (dark grey) of the fingers, for closing (light grey) respectively opening (dark grey) of the hand and precision grip, for flexion, pronation and radial inclination (light grey) respectively extension, supination and ulnar inclination (dark grey) of the wrist. C. Peak velocity of the extension phases as a function of that of the flexion phases, for each participant (different symbols) and each type of finger and hand (not wrist) phantom movements. Each symbol represents the mean value obtained by averaging over all cycles for a given type of phantom movement. The dashed black line indicates a theoretical slope of 1 . The linear regression line is traced in grey. The p-value of the Pearson test for linear correlation (interception forced at $(0,0))$ is indicated together with the linear regression equation. Note that the slope is 1.24 , meaning that extensions are on the average $24 \%$ faster than the flexion phantom finger and hand movements.

\subsection{Influence of time elapsed since amputation}

Finally, we tested whether, within our sample, characteristics of phantom movements evolved over time. Fig. 3 shows, for each patient, the number of types of phantom movements the patients could execute (Fig. 3A) as well as the mean amplitude and peak velocity (Fig. 3B) as a function of elapsed time since the amputation for those phantom movements which 
all patients could execute, i.e., thumb flexion/extension and global hand opening/closure (see Table 2). Statistical testing revealed the absence of a relation between these variables and the elapsed time (Number of movements: $\mathrm{n}=8, \mathrm{t}=1.65, \mathrm{p}>0.1$; Amplitude: $\mathrm{n}=7, \mathrm{t}=1.42, \mathrm{p}>0.2$; Peak velocity: $\mathrm{n}=7, \mathrm{t}=1.13, \mathrm{p}>0.3)$.
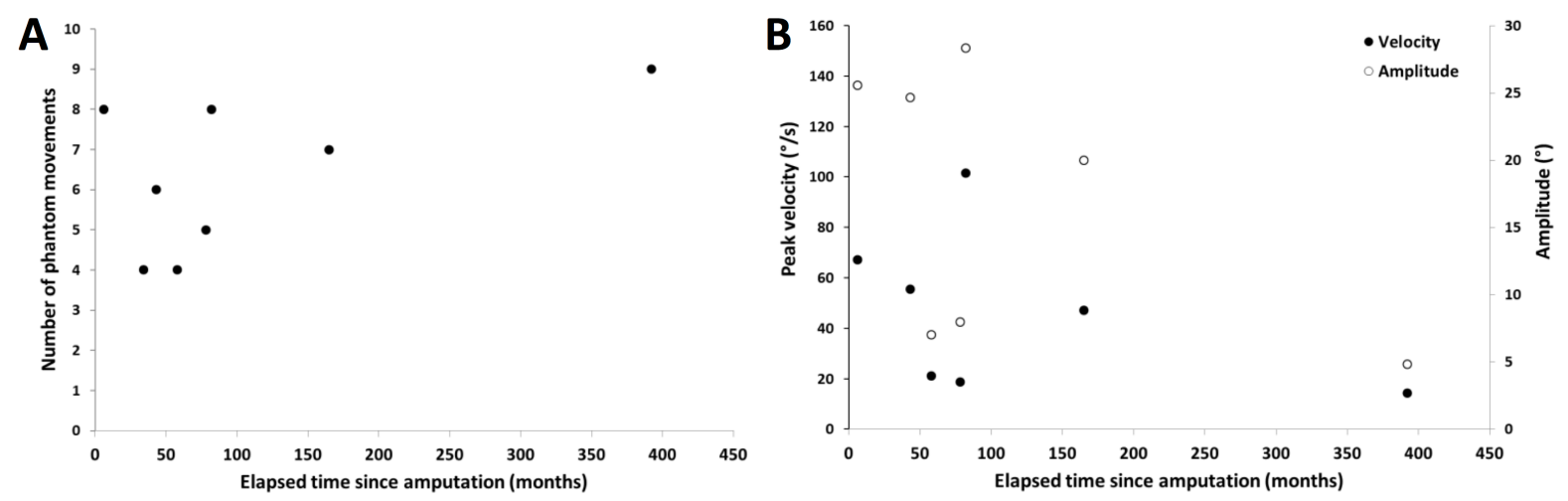

Fig. 3. (A) Number of different types of phantom finger hand and wrist movements that each patient can perform as a function of the time elapsed since amputation for all 8 patients. (B) Amplitude (open circles) respectively peak velocity (black circles) as a function of the time elapsed since amputation for 7 patients (not for P4), averaged over all cycles of phantom thumb and global hand movements. Pearson tests for linear correlation showed no significant correlation ( $\mathrm{p}>0.1$ for all three variables).

\section{Discussion}

The goal of the present study was to explore the characteristics of voluntary phantom finger, hand and wrist movements in arm amputees. The results revealed that the types of phantom movements which could be performed varied amongst the patients. Without exception, the patients felt the execution of phantom movements as "natural" but effortful. The kinematic analysis of the phantom movements showed that phantom movements were generally small and slow. Extension/opening finger, precision grip and hand movements were faster than flexion/closure movements. Finally, in our sample we found no relation between our kinematic variables and the time elapsed since the amputation. These findings are discussed below after some methodological remarks.

\subsection{Methodological remarks}

Since the kinematic analysis is entirely based on movements of the intact limb, it must be assumed that the intact limb correctly mimicked the phantom movements; if not, our interpretations will be erroneous. We feel, however, confident about this for three reasons. First, some patients asked to repeat a given condition a second time "since they had not correctly imitated the movements". This suggests that they had a good perception about both their phantom and intact hand movements. Second, the kinematic analyses showed that the phantom movements were small and slow relative to intact limb movements. For instance, the amplitude of phantom finger and hand movements, averaged over all patients, ranged from $9^{\circ}$ to $33^{\circ}$, and for wrist movements from $23^{\circ}$ to $77^{\circ}$, which is much smaller than can be performed with an intact limb. So, if the patients had not carefully imitated the phantom 
movement, one could have expected faster movements with a larger movement range. Third, for the patients that reported increasing effort and fatigue during the execution of some phantom movements, the kinematics of the intact hand became drastically reduced, both in amplitude and in frequency, before stopping. Since the actual movements of the intact hand certainly gave no rise to fatigue of that hand (given the small amplitudes, velocities and cycle frequencies), this again shows that the patients imitated their phantom movement. Therefore, we can be confident that the kinematics of the intact limb movements actually reflects the kinematics of the phantom movements.

We chose to quantify the finger and hand movements by averaging the angular values obtained for each articulation involved. We could have chosen other ways, such as only considering the channel with the largest movement range or summing-up all involved articulation angles. However, since we were interested in comparing flexion versus extension movements and in relations between different variables based on the same type of angular analysis, this choice does not influence the interpretation of the results.

\subsection{Phantom mobility}

All recorded patients were able to perform different types of phantom movements: each patient made flexion/extension movements of the thumb and could open/close their hand; all but one were able to open/close their precision grip; six of them made at least one other type of individual finger movements; and six of the patients were able to make at least one type of phantom wrist movement. The range of motion as well as the velocity of execution were highly variable (both between patients for a given phantom movement and between types of movements for a given patient) but comfortably executed phantom movements were generally slower and smaller than intact limb movements.

Despite the slowness of the phantom movements, their movement time was short compared to those found in some previous studies. Indeed, we found the average movement cycle to last $2.3 \mathrm{~s}$ whereas Gagné and colleagues (2009) found $7 \mathrm{~s}$ per cycle. Their longer movement times can neither be explained by larger movement amplitudes (as these variables were uncorrelated in their study as well as in the present one), nor by the absence of phantom pain in our patients. Indeed, in their study, the movement time of $7 \mathrm{~s}$ was found for patients without phantom pain; patients with phantom pain even showed longer movement times. Yet, Reilly and colleagues (2006) found similar movement times as those in the present study. Several reasons might be responsible for differences in movement times, such as use of myoelectric prostheses in Reilly's and the present study versus none for Gagné's study; or 5 performed movement cycles in Gagné's study versus 10 in Reilly's and the present study. But given the limited number of participants and the huge variability in movement times between participants and between types of phantom movements, it is not possible to conclude on this point.

It is interesting to note that although patient P4 was not able to individually move phantom finger 2 (index finger), he could make a precision grip (i.e., thumb/index opposition) and global hand opening/closure. P5 was unable to move index and pinkie but he performed a precision grip with a thumb/pinkie opposition as well as global hand opening/closure. Also, P8 could individually move his fingers but was unable to make a precision grip. These 
observations suggest that motor control of phantom movements involving more than one finger is not just the sum of motor control of individual fingers. Moreover, the fact that thumb movements, precision grip and global hand movements are strongly preserved in amputees, even after more than 32 years, reflects the strength of the implementation of manual motor control in the human brain. This point will feature again in the Neuroplasticity section below.

Patients generally describe that "something slows down the movement" or that they "would like to go faster but there is a resistance". Why are phantom movements felt as slow? Two non-exclusive explanations appear. First, the motor commands sent to the lost limb might be modified with respect to motor commands to intact limbs following cortical plasticity after the amputation (e.g., Gagné et al., 2011; Ramachandran et al., 2010). Yet, a typical characteristic of intact-limb motor control is preserved in phantom movements: extension/opening phases of the cyclic finger and hand movements were globally faster than flexion/closure phases. This is easy to understand for intact limb movements since flexion/closure of fingers, precision grip and hand are functionally important for grasping objects and are, therefore, more precisely controlled than extension/opening movements that usually only serve to withdraw the hand from the object. The well-known speed-accuracy trade-off principle (Fitts, 1954; Shannon and Weaver, 1949) describes that precise manual prehension movements are slower (e.g., Bootsma et al., 1994). Yet, phantom flexion/closing movements don't need to be precise: there is no object to grasp and the fingers cannot touch each other. So, the fact that phantom movement execution still shows characteristics of intact limb motor control, even after many years, suggests that, despite anatomical and functional reorganizations in the motor pathways, the motor commands for phantom movements are (at least partly) preserved.

An alternative explanation for the perceived slowness of phantom movements is related to the lack of feedback about the movement execution. In intact limb movements, proprioceptive and visual feedback inform about the joint angles, the speed of the movement and the eventual opposition to movement execution (i.e., the produced force). Microneurographic recordings from cutaneous afferents emanating from the human wrist and finger joints have shown that dorsal skin receptors can potentially provide information on movements of the hand and fingers (Edin and Johansson, 1995). In addition, both agonist and antagonist muscle spindle inputs contribute to joint movement coding (Ribot-Ciscar and Roll, 1998). But for phantom movements the only feedback comes from residual muscle contractions (Reilly et al., 2006) and some skin movement of the residual limb. Moreover, the ago-antagonist muscle spindle information is largely perturbed by the amputation (residual muscles are damaged and no longer firmly attached to the bones). For amputees this means that feedback about phantom movement execution comes from muscles and skin that were prior to the amputation not (in upper arm amputees) or only partly (in forearm amputees) involved in finger, hand, or wrist movement execution. The sensorimotor system is not used to interpreting this sensory information in relation to hand movements. Moreover, it cannot be recalibrated on the basis of visual information since there is no visual feedback about a phantom movement. The importance of visual feedback on phantom motor sensation and on residual limb muscle activity has recently been demonstrated (Imaizumi et al., 2014; 
Kawashima et al., 2013). It would be interesting to test our hypothesis with a protocol in which the patient mimics the phantom movement while looking into a mirror reflecting their intact hand at the location of their phantom hand. If our hypothesis about the lack of meaningful feedback as a cause for the slowness of phantom movements is valid, seeing the mimicking hand at the place of the phantom hand could provide meaningful visual feedback of the kinematics of their phantom movement and might increase the velocity of its execution.

The experienced fatigue related to phantom movement execution might equally be related to the lack of movement feedback. With the exception of patient P8 who was able to perform many cycles without fatigue, all patients reported a sense of effort during phantom movement execution. Their remark that they "want to go faster but there is a resistance" could be explained by the mismatch between the actual movement feedback and their expectation based on the sent motor command, which might lead to fatigue after some cycles.

\subsection{Evolution of phantom mobility}

Reilly and colleagues (2006) emphasized the precocity of the phantom limb mobility occurrence but also the tendency for the number of possible phantom movement types to diminish with time, ending sometimes in total phantom limb immobility (see also Henderson and Smyth, 1948; Hunter et al., 2008). The results of the present study suggest that neither the number of different types of phantom movements, nor kinematic variables such as movement range and peak velocity, systematically diminish over time. In addition, Henderson and Smyth (1948) had observed that, after amputation, finger flexion movements persist longer than finger extension movements. The results of our study do not confirm this: all patients who were able to perform flexion movements were also able to perform extension movements. The kinematic analysis even showed that the extension was faster than the flexion phase.

These general findings are not due to practicing, since (other than P6 for pinkie movements) our patients denied systematically practicing their phantom movements as "they are not useful". Yet, this issue seems to be important. Most patients spontaneously mentioned that their movement capacities "would have been better if they had trained the phantom movements before coming to the recording session". In this light, P4 is an interesting case. This patient had ignored his phantom limb for years. During the interview on phantom mobility he tried to move his phantom limb to see "whether he could still control it". Although the movements were extremely slow and small (as we observed from the mimicking intact hand), to his surprise, he could open and close his hand and make some thumb flexions. He promised to practice phantom hand movements and when he came back for the recording session (some weeks later) he was able to make four different phantom movements (see Table 2). Also, it happened several times during the recording sessions that a type of movement the patient had indicated as impossible became possible during the recording session, as if it was "woken up". As far as we know, this has never been reported nor studied.

\subsection{Neuroplasticity}

It is a well-established idea that the primary somatosensory and motor cortices undergo substantial reorganization after amputation. The cortical regions whose neuronal activity were related to the motor control and sensory information of the now missing limb are believed to 
gradually become active in relation to another, usually adjacent, part of the body (Cohen et al., 1991; Flor et al., 1995; Vandermeeren et al., 2003). Indeed, TMS over the former hand area of M1 evokes motor evoked potentials in residual limb muscles (Gagné et al., 2011; Mercier et al., 2006), showing that motor commands sent from the cortical hand area are now projecting on more proximal limb muscles. However, if the primary sensorimotor areas were reorganized in such a way, it should lead to disappearance of sensations from, and motor control of, the lost limb. The presence of phantom limbs and (voluntary) movements shows that this is obviously not the case. This is confirmed by the fact that TMS over the primary sensorimotor cortices, while evoking muscle activity in the residual limb muscles, actually gives rise to phantom hand sensations, including phantom movements (Mercier et al., 2006). So, (at least part of) the lost limb is still represented in the primary sensorimotor areas, suggesting that the cortical reorganization is limited. This hypothesis is reinforced by the finding that phantom limbs mostly concern the fingertips and parts around joints (Touillet et al., submitted). Indeed, being precisely controlled, these body parts have always been better represented in the primary sensorimotor cortical areas than the back of the hand or the middle of the forearm for example.

So how can it be that TMS of the hand area of the primary motor cortex activates proximal muscles? It has been shown in non-human primates that spinal hand motoneurons after axotomization by amputation can innervate remaining stump muscles ( $\mathrm{Wu}$ and Kaas, 2000; Qi et al., 2004). In that case, motor commands from the primary motor hand area can indeed activate residual limb muscles. Moreover, if afferent nerves also retarget on these muscles, a closed sensorimotor loop is preserved between muscles and cortical areas formerly related to the lost limb. This could explain the observed (phantom) movement representations (Reilly et al., 2006). Still, phantom sensations do not always go together with mobility of the phantom limb since patients can be conscious of their phantom limb but not be able to mobilize it at will (Reilly et al., 2006). So, limb movement representation does not automatically go in line with limb awareness, suggesting distinct cortical and/or neuromuscular reorganizations. What's more, distinct reorganizations of the primary motor cortex and the primary somatosensory cortex have already been found (e.g., Ojemann and Silbergeld, 1995). It would be interesting to study cortical involvement in voluntary phantom movements by brain exploration techniques such as EEG, and this from soon after amputation and onwards.

\subsection{Conclusion}

The present study showed that the execution of phantom movements is perceived as "natural" but effortful. Although phantom movements are generally smaller and slower than intact limb movements, some characteristics of intact limb motor control can still be found, suggesting that phantom motor commands are (at least partly) similar to those for intact limbs. Neither the number of types of phantom movements that can be executed nor the kinematic characteristics were related to the elapsed time since amputation, highlighting the persistence of post-amputation neural adaptation. We hypothesize that the perceived slowness of phantom movements is related to altered proprioceptive feedback that cannot be recalibrated by the lack of visual feedback during phantom movement execution. 


\section{Funding}

This project was supported by the Région Provence-Alpes-Côte d'Azur (Project ExplorAmp 2012, $n^{\circ}$ 2012_07072) and CNRS (Project RéorgAmp Defisens 2013, $\mathrm{n}^{\circ} 75114$; Defisens 2014, $\mathrm{n}^{\circ}$ 40507). The funding sources had no other involvement than providing the financial support.

\section{Acknowledgments}

We warmly thank the patients who so willingly gave us several hours of their time to teach us about phantom movements. We are also very grateful to the medical staff and the Prosthetics Department of the rehabilitation institutes who kindly received JdG, CN and NJ. We specially thank François Codemard who organized the recording sessions.

\section{Author contributions}

Conceived and designed the experiments: JdG, NJ, CN, AT. Selection of patients: AT, NM, JP, LM. Performed the experiments: JdG, NJ, CN, AT, JP. Wrote analysis tools: NJ, TC. Analyzed the data: JdG, NJ. Wrote the paper: JdG.

\section{Conflict of Interest Statement}

The authors declare that the research was conducted in the absence of any commercial or financial relationships that could be construed as a potential conflict of interest.

\section{References}

André, J.M., Paysant, J., Martinet, J., Beis, J.M. (2001). Classification and mechanisms of body perceptions in the amputees. Ann. Readapt. Med. Phys. 44, 13-18.

Bootsma, R.J., Marteniuk, R.G., MacKenzie, C.L., and Zaal, F.T.J.M. (1994). The speedaccuracy trade-off in manual prehension: effects of movement amplitude, objects size and object width on kinematic characteristics. Exp. Brain Res. 98, 535-541.

Carlen, P.L., Wall, P.D., Nadvorna, H., Steinback, T. (1978). Phantom limbs and related phenomena in recent traumatic amputations. Neurol. 28, 211-217.

Cohen, L.G., Bandinelli, S., Findley, T.W., Hallett, M. (1991). Motor reorganization after upper limb amputation in man. A study with focal magnetic stimulation. Brain 114, 615627.

Edin, B.B., and Johansson, N. (1995). Skin strain patterns provide kinesthetic information to the human contral nervous system. J. Physiol. (London) 487, 243-251.

Fitts, P.M. (1954). The information capacity of the human motor system in controlling the amplitude of movement. J. Exp. Psychol. 47, 381-391. 
Flor, H., Elbert, T., Knecht, S., Wienbruch, C., Pantev, C., Birbaumer, N. et al. (1995). Phantom-limb pain as a perceptual correlate of cortical reorganization following arm amputation. Nature 375, 482-484.

Gagné, M., Reilly, K.T., Hétu, S., Mercier, C. (2009). Motor control over the phantom limb in above-elbow amputees and its relationship with phantom limb pain. Neurosci 162, 78-86.

Gagné, M., Hétu, S., Reilly, K.T., Mercier, C. (2011). The map is not the territory: Motor system reorganization in upper limb amputees. Hum. Brain Mapp. 32, 509-519.

Henderson W.R., and Smyth G.E. (1948). Phantom limbs. J. Neurol. Neurosurg. Psych. 11, 88-112.

Huenerfauth, M., and Lu, P. (2010). Accurate and accessible motion-capture glove calibration for sign language data collection. ACM Transactions on Accessible Computing (TACCESS) 3(1). DOI 10.1145/1838562.1838564.

Hunter, J.P., Katz, J., Davis, K.D. (2008). Stability of phantom limb phenomena after upper limb amputation: A longitudinal study. Neurosci. 158, 939-949.

Imaizumi S., Asai, T., Kanayama, N., Kawamura, M., Koyama, S. (2014). Agency over a phantom limb and electromyographic activity on the stump depend on visuomotor synchrony: a case study. Front. Hum. Neurosci. 8, article 545. Doi: 10.3389/fnhum.2014.00545.

Kawashima, N., Mita, T., Yoshikawa, M. (2013). Inter-individual difference in the effect of morror reflection-induced visual feedback on phantom limb awareness in forearm amputees. PLOS one 8(7), e69324.

Kooijman, C.M., Dijkstra, P.U., Geertzen, J.H.B., Elzinga, A., Van der Schans, C.P. (2000). Phantom pain and phantom sensations in upper limb amputees: an epidemioligical study. Pain 87, 33-41.

Mercier, C., Reilly, K.T., Vargas, C.D., Aballea, A., Sirigu, A. (2006). Mapping phantom movement representations in the motor cortex of amputees. Brain 129, 2202-2210.

Mitchell, S.W. (1871). Phantom limbs. Lippincott's magazine 8, 563-569.

Ojemann, J.G., and Silbergeld, D.L. (1995). Cortical stimulation mapping of phantom limb rolandic cortex. Case report. J Neurosurg 82, 641-644.

Qi, H.X., Stewart, P.W., Kaas, J.H. (2004). Connections of neurons in the lumbar ventral horn of spinal cord are altered after long-standing limb loss in a macaque monkey. Somatosen Mot Res 21, 229-239.

Raffin, E., Giraux, P., Reilly, K.T. (2012a). The moving phantom: Motor execution or motor imagery? Cortex 48, 746-757.

Raffin, E., Mattout, J., Reilly, K.T., Giraux, P. (2012b). Disentangling motor execution from motor imagery with the phantom limb. Brain 135, 582-595.

Ramachandran, V.S., and Hirstein, W. (1998). The perception of phantom limbs. The D.O. Hebb lecture. Brain 121, 1603-1630.

Ramachandran, V.S., Brang, D., McGeoch, P.D. (2010) Dynamic reorganization of referred sensations by movements of phantom limbs. NeuroReport 21, 727-730

Reilly, K.T., Mercier, C., Schieber, M.H., Sirigu, A. (2006). Persistent hand motor commands in the amputees. Brain 129, 2211-2223. 
Ribot-Ciscar, E., Roll, J.P. (1998). Ago-antagonist muscle spindle inputs contribute together to joint movement coding in man. Brain Res. 791, 167-584.

Schott, G.D. (2014). Revealing the invisible: the paradox of picturing a phantom limb. Brain 137, 960-969.

Shannon, C.E., Weaver, W. (1949). The Mathematical Theory of Communication. Urbana, IL: University of Illinois Press.

Vandermeeren, Y., Bastings, E., Good, D., Rouiller, E., Olivier, E. (2003). Plasticity of motor maps in primates: recent advances and therapeutical perspectives. Rev Neurol (Paris)159, 259-275.

Wu, C.W., Kaas, J.H. (2000). Spinal cord atrophy and reorganization of motoneuron connections following long-standing limb loss in primates. Neuron 28, 967-978.

Xerri, C. (2003). Plasticité des représentations somesthésiques et illusions perceptives: le paradoxe du membre phantôme. Intellectica 36-37, 67-87. 